\title{
Cancer Risk Assessment of Patients Undergoing Computed Tomography Examination at the Korle-Bu Teaching Hospital
}

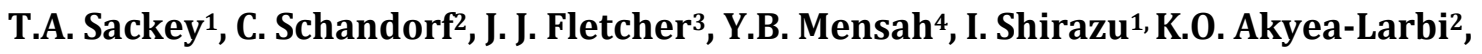 \\ E. K. Tiburu ${ }^{5}$, S.T. Odonkor ${ }^{1}$ \\ ${ }^{1}$ Radiological and Medical Sciences Research Institute, Ghana Atomic Energy Commission, Accra-Ghana. \\ ${ }^{2}$ School of Nuclear and Allied Sciences, University of Ghana, Accra-Ghana. \\ ${ }^{3}$ Department of Applied Physics, University of Development Studies, Navrongo-Ghana \\ ${ }^{4}$ Radiology Department, Korle-Bu Teaching Hospital, Accra-Ghana \\ 5Department of Biomedical Engineering, University of Ghana, Legon-Accra
}

\section{ABSTRACT}

The aim of this study is to estimate the effective dose and assess the lifetime attributable risk of cancer incidence of patients undergoing computed tomography scan at the Korle-Bu Teaching Hospital in the Greater Accra Region of Ghana. Data on Volume CT Dose Index (CTDI $\left.{ }_{\mathrm{vol}}\right)$ and Dose Length Product (DLP) displayed on the scanner control console was recorded after confirmation of the results by performing independent checks on a phantom. The effective doses were estimated using the DLP displayed and the anatomic region specific

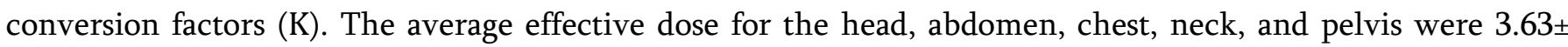
$2.39 \mathrm{mSv}, 15.37 \pm 8.49 \mathrm{mSv}, 12.72 \pm 13.97 \mathrm{mSv}, 4.04 \pm 1.47 \mathrm{mSv}$ and $15.8 \pm 3.59 \mathrm{mSv}$ respectively. Effective doses for the head and neck were within the typical range of $(1-10 \mathrm{mSv})$ for CT examinations whilst abdomen, chest and pelvis were above $10 \mathrm{mSv}$. The average life attributable risk of cancer incidence for each region of examination were determined from the effective dose, sex and age using the model proposed in BEIR VII Report . The average cancer risk incidence for head, neck, chest, abdomen and pelvis examinations were low in the range 1 in 10,000 to 1 in 1,000. There were wide variations in the effective dose values obtained for the same region under examination. This trend calls for the optimization of CT examination protocols to be established to ensure that patient doses are as low as reasonably achievable, economic and social factors being taken into account especially for chest examinations.

Keywords: Computed Tomography, Dose Length Product, Effective Dose, Cancer Risk, Cancer Incidence, Life Attributable Risk

\section{INTRODUCTION}

There has been a sharp increase in CT examination in recent times. There are approximately 80 million CT examinations performed in the United States of America annually of which about 7 million occur in children [1]. In Ghana, it is estimated that between 40,000 and 55,000 go for CT examinations in a year [2]. Even though the dose received from such an examination is small compared to Radiotherapy procedures and so the deterministic effect is minimal.
The increasing number of the population undergoing the CT examination gives cause for concern when one considers stochastic effects and therefore the number of people who risk developing cancer. CT is the most substantial contributor to the collective effective dose for all radiographic procedures [3]. Surveys [4-8] have shown that the contribution of CT to the total collective dose can be as high as $67 \%$ in the United States and $40 \%$ in Europe although CT examinations represent only $11 \%$ and $4 \%$ respectively. Therefore, the dose levels delivered in CT examinations should 
be known and available to patients and their physicians. Also, the rapid evolution of CT technology and the resultant explosion in new clinical applications including cardiac CT combined with the significance of CT dose levels and the CT contribution to the collective dose to the population have created a compelling need to understand and to reduce dose that the patients receive especially the pediatric patients [9]. In other words one has to Image gently.

Computed Tomography has become a routine imaging modality for many clinical applications due to its wide availability, less invasiveness, short scanning time, excellent anatomical resolution and high diagnostic value [10]. It is also suitable for patients who are contraindicated for magnetic resonance imaging procedures. In Ghana there is an increase in CT applications as more CT centres are being established [2]. But CT examination is known to give much higher doses compared with other diagnostic imaging techniques; therefore, CT patient dose management systems must be optimized. The increasing numbers of the population undergoing the CT examination gives cause for concern when one considers the stochastic effects and therefore the number of people who risk developing cancer. Again, large variations in exposure parameters and patient doses even for a single CT examination have been reported [11]. There is therefore the need to determine the effective doses of patients who undergo CT examination and hence to be able to estimate the cancer risk and the life attributable risk to serve as a guide to the hospital staff in charge of the CT examinations [12].

In estimating lifetime risks of cancer incidence and mortality, models are developed and used. These models take into account sex, age at exposure, dose rate and other factors [13]. Estimates are given for all solid cancers, leukaemia and cancer of several specific sites. Most of these risks models are based on data from Japanese atomic bomb survivors. Risk estimates are subject to several sources of uncertainty due to inherent limitations in epidemiology data. In addition to statistical uncertainty, the populations and exposures for which risk estimates are needed nearly always differ from those from whom epidemiology data are available [12]. As indicated much earlier, risk may depend on the type of cancer, the magnitude of the dose, the quality of the radiation, the dose - rate, the age and sex of the person exposed, exposure to other carcinogens such as tobacco, and other characteristics of the exposed individual. In recent times, several national and international organizations have developed models for estimating cancer risk from exposure to low levels of low LET ionizing radiation. These bodies include Biological Effect of Ionizing Radiation (BEIR V) committee in 1990, the International Commission on Radiological Protection (ICRP, 2007) [14], the National Council on Radiation Protection and measurements (NCRP, 1993) [15], the United State Environmental Protection Agency (EPA, 1994, 1999) [16], the United Nations Scientific Committee on the Effect of Atomic Radiation (UNSCEAR, 2000) [ 17]. But by far, the body which have been accepted and quoted extensively in literature for cancer risk estimation is Biological Effect of Ionizing Radiation (BEIR VII) report of 2006. BEIR VII (2006) report provides a method for the estimation of Lifetime Attributable Risk (LAR) of Cancer based on the magnitude of a single radiation exposure and a patient's age at exposure [13].

\section{Aim}

The main objective of this work is to determine the effective dose of patients undergoing Computed Tomography at the Korle-Bu Teaching Hospital and secondly, assess the risk of developing cancer in the future of such patients.

\section{MATERIALS}

The materials used for this study include:

$\checkmark \quad 640$ - slice Toshiba CT scanner (Aquilion One).

$\checkmark$ Barracuda multipurpose multi-meter. 
$\checkmark$ CTDI head phantom

$\checkmark$ CTDI body phantom.

$\checkmark$ Microsoft excels spreadsheet and access database tools.

$\checkmark$ Water phantom for checking resolution.

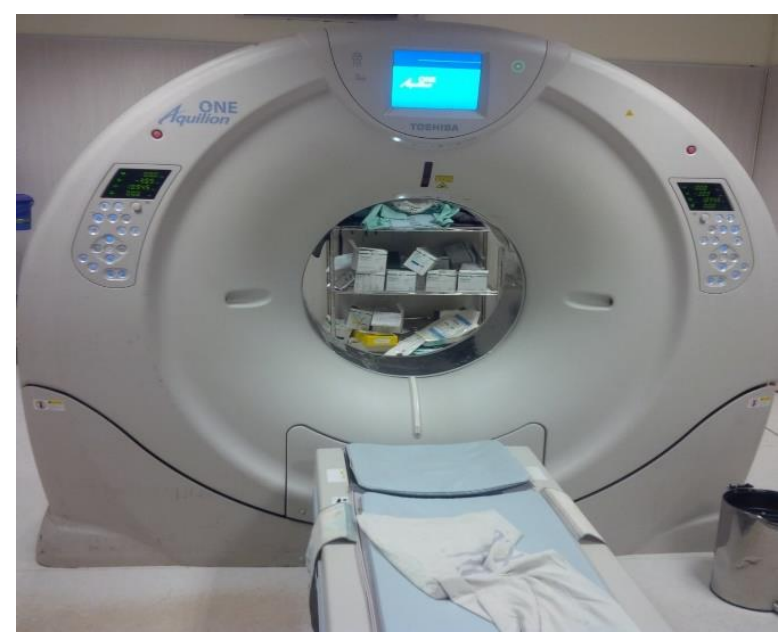

Figure 1. Aquilion One CT Scanner

\section{METHODOLOGY}

\section{Image Data Collection}

The Advanced Medical Imaging Centre which houses the Toshiba Aquilion One CT scanner has been in operation since December 2012.Three Hundred and (300) patients were randomly selected for this study. Parameters such as; $\mathrm{kVp}$, mAs, DLP, CTDIvol, Pitch factor and scan length, were collected from the control console, matching each patients identity with a given serial number with the given parameters. Data was reduced and analysed with the Microsoft Excel 14.0.

\section{1) Estimation of Effective Dose}

The effective dose was estimated using the equation 1 .

$E=D L P \times K$ 1

where, $K$ is the slope of the $E$ and the DLP relationship, the $K$ values varies for various CT examinations. These are given in table 1 for adults examinations at $120 \mathrm{kV}$.
Table 1. Conversion factors from DLP to effective Dose

\begin{tabular}{|c|c|}
\hline $\begin{array}{l}\text { TISSUE } \\
\text { ORGAN }\end{array}$ & $\begin{array}{l}\text { CONVERSION } \\
\text { FACTOR } \boldsymbol{K}(E / D L P) \\
{[\mathrm{mSv} / \mathrm{mG} y-\mathrm{cm}]}\end{array}$ \\
\hline Head & 0.0019 \\
\hline Neck & 0.0051 \\
\hline Chest & 0.0145 \\
\hline Abdomen & 0.0153 \\
\hline Pelvis & 0.0129 \\
\hline
\end{tabular}

\section{Estimation of Lar of Cancer}

The LAR is defined as additional cancer risk above and beyond baseline cancer risk and can be calculated for specific cancers as well as for all cancers combined [18].

The LAR was calculated using the equation 2.

BEIR VII LAR at an age $=$

$\left(\frac{\mathrm{E}(\mathrm{mSv})}{\mathrm{D}} \times \frac{\mathrm{LAR}(\text { cancer incidence })}{100,000}\right) \times 100 \%$

$\mathrm{D}=0.1 \mathrm{~Gy}$, the reference dose to the population considered in the BEIR VII report.

\section{RESULTS AND DISCUSSION}

\section{Presentation of Results}

The results of the measurements are presented in Table 2

Table 2: QC Measurements

\begin{tabular}{|l|l|l|}
\hline Parameter & $\begin{array}{l}\text { Deviation of } \\
\text { X-ray machine } \\
\text { (measurement) }\end{array}$ & $\begin{array}{l}\text { Acceptable } \\
\text { Deviation } \\
\text { (Value) }\end{array}$ \\
\hline KV Accuracy & $2.4 \%$ & $\leq \pm 6.0 \%$ \\
\hline HVL@130KV & 7.85 & $\geq 3.2$ \\
\hline
\end{tabular}


Table 3 Summary for CTDI test

\begin{tabular}{|l|l|l|l|}
\hline $\mathbf{k V}$ & Phantom Type & CTDIvol (mGy) & DLP (mGycm) \\
\hline 110 & Head & 29.82 & 447.3 \\
\hline 130 & Head & 43.95 & 659.3 \\
\hline 110 & Body & 17.75 & 266.3 \\
\hline 130 & Body & 27.50 & 412.5 \\
\hline
\end{tabular}

Table 4. Summary for Geometric Efficiency test

\begin{tabular}{|l|l|l|l|}
\hline $\mathbf{k V}$ & $\begin{array}{l}\text { Geometric } \\
\text { Efficiency }\end{array}$ & $\begin{array}{l}\text { Beam width } \\
\text { (FWHM) }\end{array}$ & $\begin{array}{l}\text { CTDI (100) } \\
\text { (mGycm) }\end{array}$ \\
\hline 80 & $91.6 \%$ & $11.1 \mathrm{~mm}$ & 26.18 \\
\hline 110 & $91.5 \%$ & $11.1 \mathrm{~mm}$ & 48.77 \\
\hline 130 & $89.2 \%$ & $11.1 \mathrm{~mm}$ & 69.96 \\
\hline
\end{tabular}

Table 5. CTDIvol and DLP Reference levels.

\begin{tabular}{|c|c|c|c|c|c|c|}
\hline \multirow[t]{2}{*}{ Examination } & \multicolumn{4}{|c|}{ National Reference Levels } & \multicolumn{2}{|c|}{ This Study } \\
\hline & $\begin{array}{l}\text { CTDI }_{\text {VoL }} \\
\text { (mGy) }\end{array}$ & \begin{tabular}{|l|} 
DLP \\
$(\mathrm{mGycm})$
\end{tabular} & \begin{tabular}{|l|} 
CTDI $_{\text {YoL }}$ \\
(mGy)
\end{tabular} & $\begin{array}{l}\text { DLP } \\
(\mathrm{mGycm})\end{array}$ & \begin{tabular}{|l|}
$\mathrm{CTDI}_{\mathrm{VOL}}$ \\
(mGy)
\end{tabular} & $\begin{array}{l}\text { DLP } \\
\text { (mGycm) }\end{array}$ \\
\hline Head (Adult) & $75^{*}$ & 930*** & $65-100 * *$ & $14^{* *}$ & 38.9 & 583.4 \\
\hline Body (Adult) & $25 *$ & $470 * * *$ & $15^{* *}$ & $700 * *$ & 15.6 & \begin{tabular}{|l|}
233.7 \\
\end{tabular} \\
\hline
\end{tabular}

${ }^{*}$ ACR ${ }^{* *}$ Netherlands, ${ }^{* * *} \mathrm{UK}$

The results obtained from the quality control measurements taken on the CT scanner to ensure the reliability of the patients' data collected on the control console. The effective dose and subsequently the lifetime cancer risk incidence of the patients undergoing the CT examinations were determined and the findings discussed.

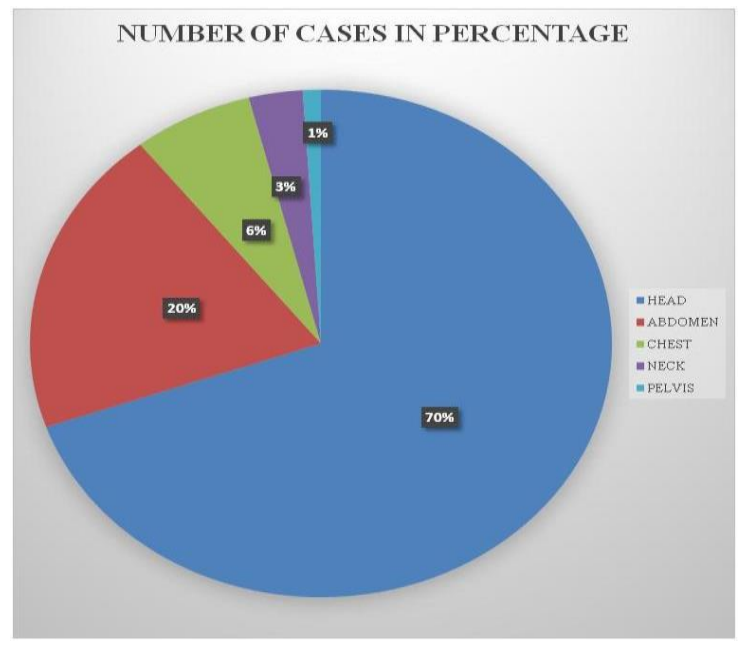

Figure 2. Pie chart of the frequency of examinations

\section{Head CT Examinations}

Three main reasons inform the choice of patients seeking any form of imaging modality. Firstly, the cost, then availability and lastly the reason for the examination. The head CT scan examination was the most frequent requested examination $(70 \%)$ at the Korle $\mathrm{Bu}$ Teaching Hospital. This is because Ultrasound Imaging which is less expensive cannot be used to image the head and conventional X-ray cannot give cross sectional images. Also, the CT scan of the head is very important for the management of Cerebrovascular accidents, Meningitis, Road Traffic Accidents, Head injuries and for staging of cancers of the head and Neck. Of the 209 cases for the Head CT, 128 were males and 81 were females (Figure 3 ). 


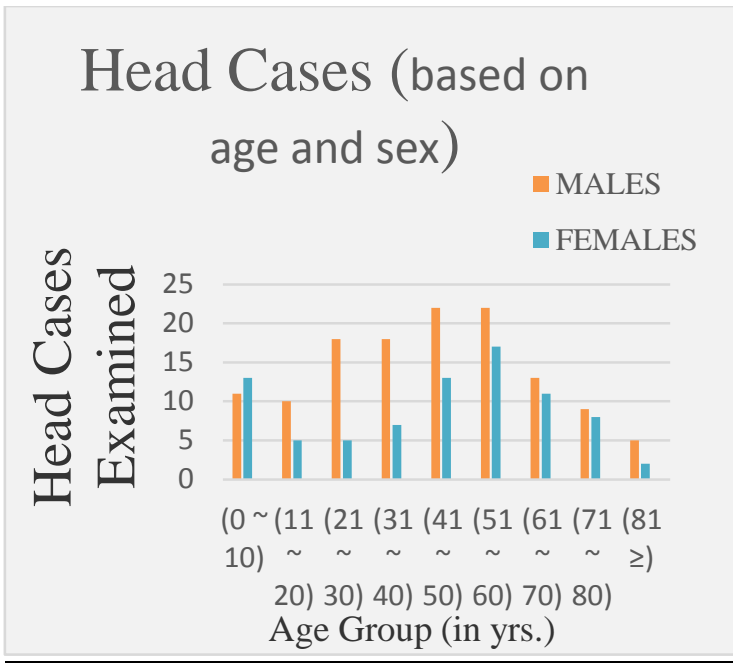

Figure 3. Head scan based on sex and age

\section{Abdomen CT Examinations}

Most of the abdominal cases rely on the Ultrasound as the main imaging modality because it is readily available, less expensive and does not give ionizing radiation. CT scan is recommended only when the ultrasound images do not show any clear cut diagnosis or when the patient cannot co-operate with the Ultrasound. Again, when cancer has to be staged and also when there are gases in the bowel then CT scan is recommended. 59 cases were recorded for the CT Abdomen which represents $20 \%$ of the sample size of which 32 were males and 27 were females (Figure 4).

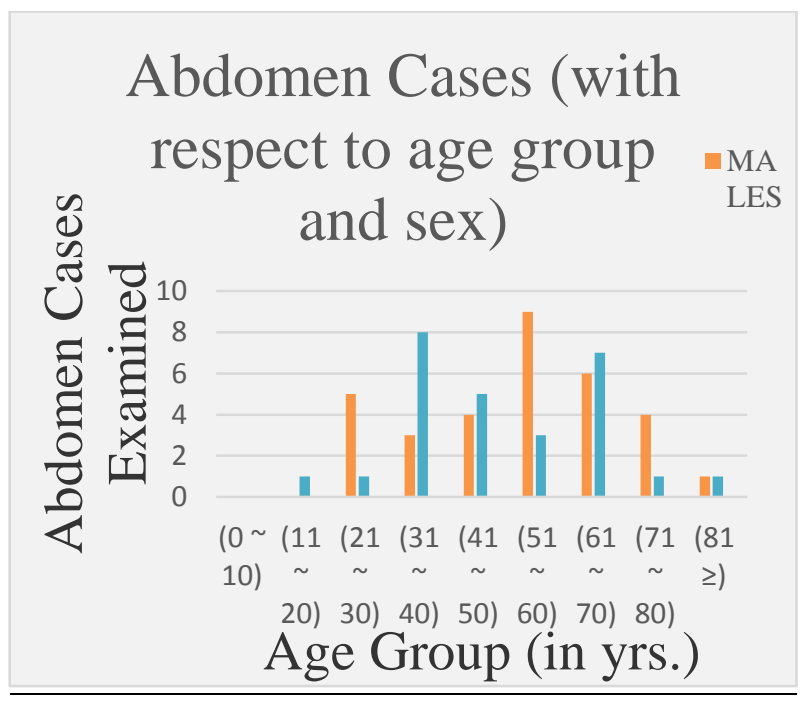

Figure 4. Abdominal scan based on sex and age

\section{Chest CT Examinations}

The chest cases seen were 20 which represent $7 \%$ of the 300 cases seen. And of the 20 cases seen, 12 were males and 8 were females. The chest CT scan examination was very few because conventional X-ray gives a lot of information in the chest such as cardiomegaly and other heart related cases. And because conventional $\mathrm{x}$-rays are cheaper may people go for that modality (Figure 5)

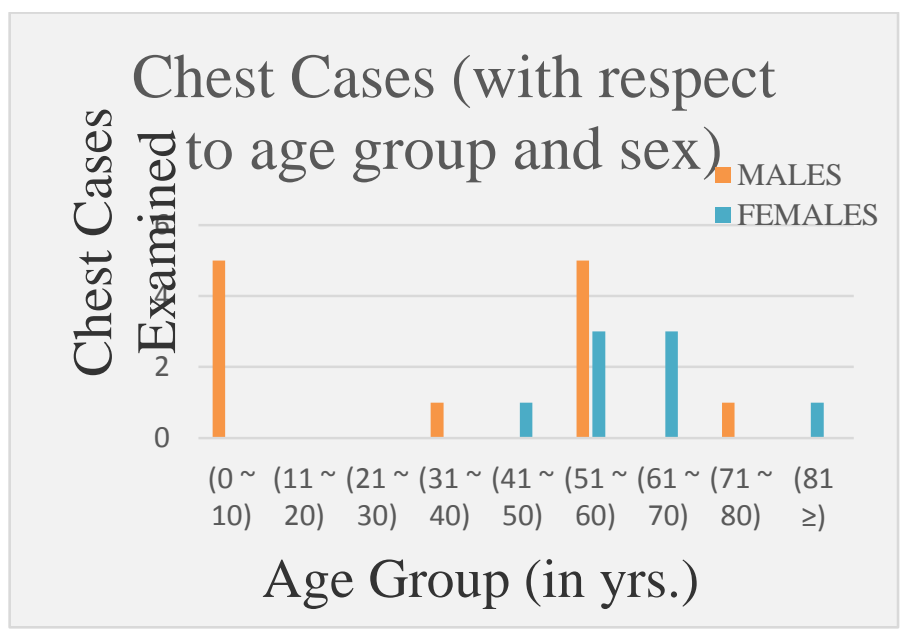

Figure 5. Histogram of chest scan by age and sex

\section{Neck CT Examinations}

There were only 8 neck cases recorded representing $3 \%$. Of this number 5 were males and 3 were females. There were only few neck cases seen (Figure 6). There were only few neck cases seen because most of the anterior neck diagnosis such as the cases involving the Thyroid gland can be picked up by Ultrasound imaging and so many patients opt for ultrasound imaging. And for the posterior, they go for the MRI since the spine is very sensitive to radiation. 


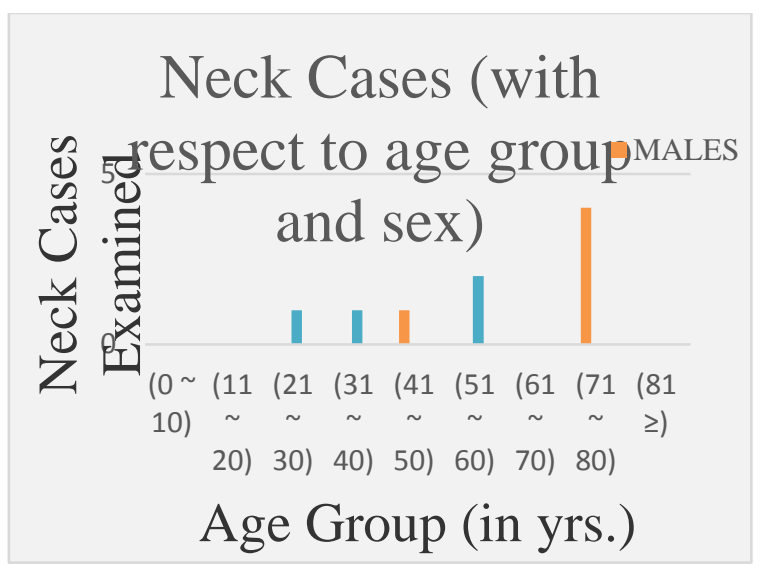

Figure 6. Histogram of Neck scan by age and sex.

\section{Pelvis CT Examinations}

There were only 3 pelvis examinations recorded representing $1 \%$. Of this number there was 1 male and 2 females (Figure 7).

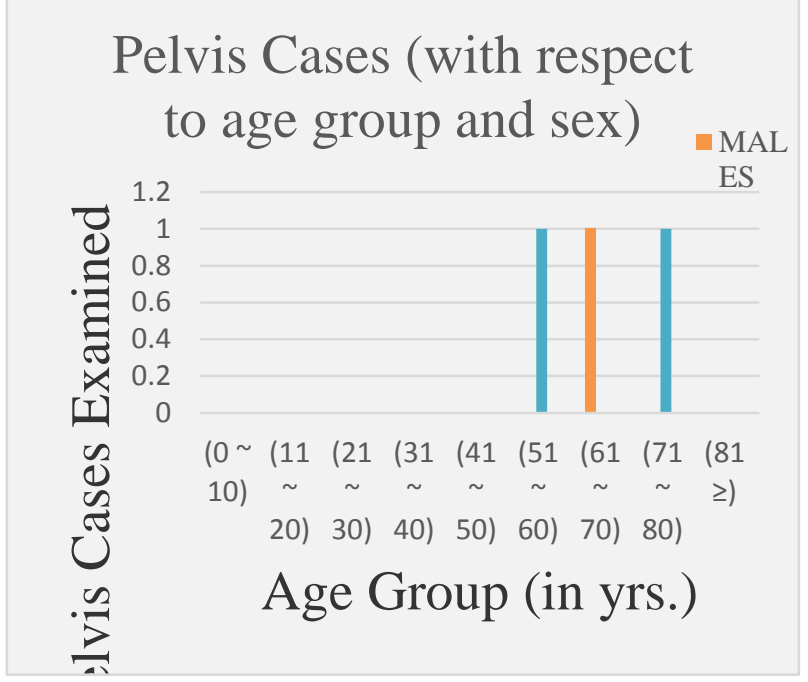

Figure 7: Bar Chart of pelvis scan by age and sex Distribution Of Ct Scans By Age

The most dorminant age group was between 51-60 years. And the youngest to be scanned was 1 day old and the oldest being 91 years. (Figure 8)

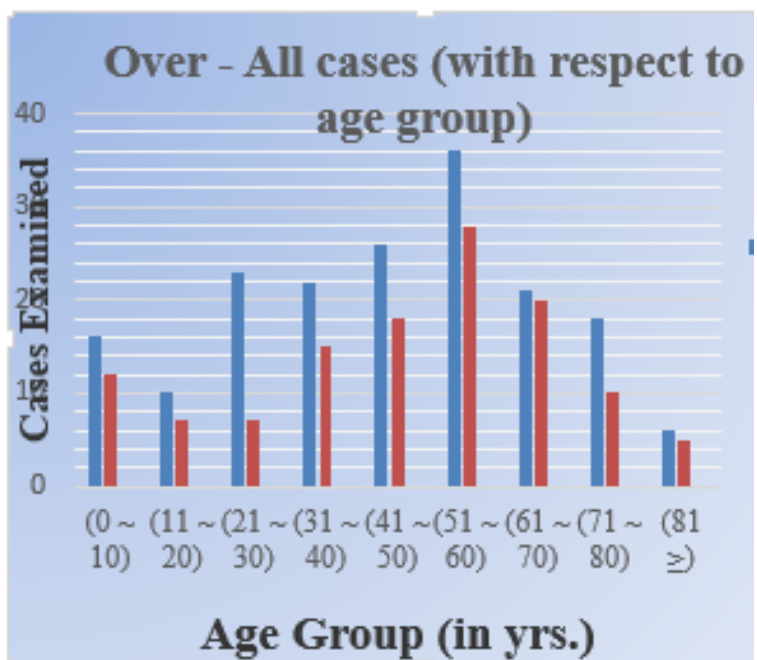

Figure 8: Histogram for all scan by age and sex

\section{Discussion}

In this research work the effective dose and the cancer risk were estimated for patients undergoing the most common types of CT examinations namely head, abdomen, chest, neck and pelvis case at the Korle-Bu Teaching Hospital. The head CT examination (70 \%) was the most frequently requested for, followed by abdomen (20\%) then chest (7\%), neck (3\%) and pelvis case representing $(1 \%)$.

There were effective dose variations depending upon the anatomic region scanned. For instance with the head, the average effective dose for the males (128 cases) was $3.622 \mathrm{mSv}$ but varied from $0.691 \mathrm{mSv}$ to $12.791 \mathrm{mSv}$ and the average for the female was $3.65 \mathrm{mSv}$ also ranging from $0.234 \mathrm{mSv}$ to $11.687 \mathrm{mSv}$. For the abdomen, the effective dose for the females varied from $4.985 \mathrm{mSv}$ to $45.86 \mathrm{mSv}$ and has an average value of $16.08 \mathrm{mSv}$ whereas for the males the effective dose varied from $4.16 \mathrm{mSv}$ to $33.78 \mathrm{mSv}$ having an average reading for the males as $14.78 \mathrm{mSv}$. The chest recorded the one with greatest variation in the effective dose values. The average effective dose for the females was $21.21 \mathrm{mSv}$ but varied from $5.80 \mathrm{mSv}$ to62.61 $\mathrm{mSv}$, whereas the effective dose for the males varied from $0.545 \mathrm{mSv}$ to $18.76 \mathrm{mSv}$ with an average effective dose of7.062 $\mathrm{mSv}$. There were only 3 pelvic cases recorded 
with the average effective dose of $15.89 \mathrm{mSv}$ but ranges from $12.345 \mathrm{mSv}$ to19.53 $\mathrm{mSv}$. The neck has mean effective dose of $4.1 \mathrm{mSv}$ and in the range of $2.568 \mathrm{mSv}$ and $5.995 \mathrm{mSv}$. The estimated average effective dose for head and neck were within the range of typical effective doses for $\mathrm{CT}$ examinations set by the European Commission (EC) and the American College of Radiology (ACR) which is $(1 \mathrm{mSv}-10 \mathrm{mSv})$. The values for the abdomen, pelvis and chest were higher than the range given. Although the effective doses recorded may look slightly higher, the study found that the lifetime attributable risk (LAR) of cancer incidence for someone who had say head scan ranges from $0.003 \%$ representing ( 1 in 33,333) and $0.078 \%(1$ in 1,280$)$.

For those who went for the abdomen scan their lifetime attributable risk of cancer incidence (LAR) was in the range of $0.021 \%$ ( 1 in 4760 ) to $0.22 \%$ ( 1 in $450)$ and for the person who had the chest scan the lifetime attributable risk of cancer incidence was in the range of $0.013 \%$ ( 1 in 7690 ) to $0.35 \%$ ( 1 in 300). So comparing these Lifetime Attributable Risk of cancer incidence (LAR) for a sample size of 300, it indicates that the patients were exposed at an acceptable risk. The average risk for all the examination was observed to be low (1 in 10000 to 1 in 100). Since stochastic risk is proportional to effective dose. Any reduction in dose will reduce the risk of having cancer. All attempts must therefore be made at all times to reduce dose to patients and therefore minimize cancer risk without sacrificing image quality.

\section{CONCLUSION}

There were wide variations in the effective dose values obtained for the same region under examination. This trend calls for the optimization of CT examination protocols to be established to ensure that patient doses are as low as reasonably achievable, economic and social factors being taken into account especially for chest examinations. The average cancer risk incidence for head, neck, chest, abdomen and pelvis examinations were low in the range 1 in 10,000 to 1 in 1,000 . The risk incurred by patients during the CT examinations were acceptable.

\section{RECOMMENDATIONS}

It is recommended that a multidisciplinary approach to patient dose management be implemented. Secondly, there should be a CT examination protocol as to what parameters to choose and who should be given contrast and who should not be given contrast. Regular training of imaging staff and referring physicians can go a long way in helping with the optimization of patient protection during the CT examinations. There is the need to established baseline data for future referencing.

\section{ACKNOWLEDGEMENT}

Thanks to the entire staff of the Medical Radiation Physics Centre/Radiological and Medical Sciences Research Institute/Ghana Atomic Energy Commission, Department of Radiology, Korle-Bu Teaching Hospital and School of Nuclear and Allied Sciences for their guidance and support.

\section{REFERENCES}

[1]. American Association of Physicist in Medicine (AAPM) Report No. 204. "Size -Specific Dose Estimation (SSDE) in Pediatric and Adult Body CT Examinations"

[2]. S. Inkoom et al, "Adult Medical X-ray Dose assessments for Computed Tomography procedures in Ghana-A review paper", Journal of Applied Science and Technology, Vol.19,No. 1 and $2: 1-9,2014$

[3]. Paul D. Deak et al, Multisection CT Protocols: Sex and Age-Specific Conversion Factors used to determine Effective Dose from Dose-Length 
Product, Radiology: Volume 257; Number 1October 2010

[4]. Federal office for Radiation Protection. Annual Report 2005. Langenhagen, Germany: Schliitersche Druck. 2005. http:// www.bfs.pdf. May 4, 2014

[5]. Brix G. Nagel HD, Stamm G, et al. Radiation exposure in multi-slice versus single slice spiral CT: result of a nationwide survey. EurRadiol 2003; 13(8): 1979-1991

[6]. Mettler FA Jr, Wiest PW, Locken J.A, Kelsey CA. CT scanning: Patterns of use and dose J Radiol Prot 2000:20(4) : 353-359

[7]. Shrimpton PC, Edyvean S. CT scanner dosimetry. Br J Radiol 1998; 71(841):1- 3

[8]. Brenner DJ, Hall EJ, Computed tomography: an increasing source of radiation exposure. N Engl T Med 2007; 357(22); 2277 - 2284

[9]. AAPM Report No.96"The Measurement, Reporting and management of Radiation Dose in CT " American Association of Physicist in Medicine ,One Physics Ellipse ,College Park , MD 207-3846 . ( 2008)

[10]. Even-Sapir E. et al. imaging of malignant bone involvement by morphologic, scintigraphic, and hybrid modalities. J Nuc Med 2005; 46(8); 135667

[11]. Mettler et al, 2000. CT scanning: Patterns of use and dose J Radiol Prot. 20(4) 353-359

[12]. Richard R. Monson et al, 'Health Risks from Exposure to Low Levels of Ionizing Radiation'; BEIR VII Report Pg 259-267 (2006)

[13]. Board of Radiation Effects Research Division on Earth and Life Sciences National Research Council of the National Academics. Health Risks from Exposure to Low Levels of Ionizing Radiation; BEIR VII Phase 2. Washington, DC: National Academies Press; 2006

[14]. ICRP. The 2007 Recommendations of the International Commission on Radiological Protection. ICRP Publication 103. Ann. ICRP. 2007; 37 (2-4): 1-332
[15]. NCRP (National Council on Radiation Protection and Measurements), 1993, Risk Estimates for Radiation Protection, NCRP Report No. 115, Bethesda, Maryland, December 31. ISBN 0-929600-34-7

[16]. Wilson, W. Design of exposure measurements for epidemiologic studies. Presented at Annual Meeting of the Chinese Assoc. for Aerosol Research, Taiwan, Taipei, October 30-31, 1999.

[17]. UNSCEAR 2000 report vol. i, sources and effects of ionizing radiation. Scientific committee on the effects of atomic radiation UNSCEAR 2000 report to the general assembly, with scientific annexes.

[18]. Rebecca Smith-Bindman et al Radiation Dose Association with Common Computed Tomography Examinations and the Associated Attributable Risk of Cancer, Arch Intern MED. VOL 169 (22) : Dec 14/28, 2009 pg 2078-2086. 